\title{
Komplizierte Ziffernkombi bei älteren Patienten
}

_ Chronische Erkrankungen treten naturgemäß eher im fortgeschrittenen Alter auf und können zur Notwendigkeit einer Palliativversorgung führen. Der EBM beinhaltet für die notwendigen Leistungen spezielle Abrechnungspositionen. Leider sind diese aber mit einer recht unübersichtlichen Zahl von Ausschlussregelungen versehen. Was also kann z. B. bei einem Patienten im 75. Lebensjahr mit den Diagnosen Diabetes mellitus, Hypertonie und Herzinsuffizienz berechnet werden, wenn er nach einem Hirninfarkt zum Palliativfall wird? Hier wird es kompliziert, denn der Patient erfüllt gleich mehrere Definitionen: Er ist chronisch krank, geriatrischer Patient und Palliativpatient.

\section{MMW-KOMMENTAR}

Die "Chronikerziffern" 03220 und 03221 EBM können auf der Grundlage der genannten Diagnosen berechnet werden, wenn mindestens zwei Arzt-Patienten-Kontakte in dem betreffenden Quartal stattgefunden haben. In gleicher Sitzung können damit die geriatrischen Leistungen nach den Nrn. 03360 bzw. 03362 kombiniert werden. Allerdings ist auch hier zu beachten, dass die Nr. 03362 nur in einer weiteren Sitzung nach dem Erstkontakt im Quartal berechnungsfähig ist. Dann wäre aber eine Kombination der Nrn. 03360 und 03362 in einer solchen (weiteren) Sitzung

\section{Tab. 1 Beispiel für die Ziffernkombination bei älteren Patienten}

\begin{tabular}{|c|c|c|c|}
\hline EBM & Legende & Euro & Bemerkungen zur Abrechnung \\
\hline $\begin{array}{l}03220 \\
03221\end{array}$ & $\begin{array}{l}\text { Behandlung und Betreuung mindes- } \\
\text { tens einer lebensverändernden chro- } \\
\text { nischen Erkrankung }\end{array}$ & $\begin{array}{r}13,57 \\
4,17\end{array}$ & $\begin{array}{l}\text { an unterschiedlichen Tagen berech- } \\
\text { nungsfähig, da zwei Arzt-Patienten- } \\
\text { Kontakte notwendig sind }\end{array}$ \\
\hline $\begin{array}{l}03360 \\
03362\end{array}$ & $\begin{array}{l}\text { Hausärztlich-geriatrisches Basis- } \\
\text { assessment und Betreuungskomplex }\end{array}$ & $\begin{array}{l}12,73 \\
16,59\end{array}$ & $\begin{array}{l}\text { in gleicher Sitzung mit den } \\
\text { Nrn. 03220/03221 kombinierbar }\end{array}$ \\
\hline 03370 & $\begin{array}{l}\text { Palliativmedizinische Ersterhebung } \\
\text { des Patientenstatus }\end{array}$ & 35,59 & $\begin{array}{l}\text { nur einmal im Jahr berechnungsfähig, } \\
\text { nicht in gleicher Sitzung mit den Nrn. } \\
\text { 03220/03221 bzw. 03360/03362 - } \\
\text { wohl aber an einem anderen Tag }\end{array}$ \\
\hline $\begin{array}{l}03371 \\
03372 \\
03373\end{array}$ & $\begin{array}{l}\text { Palliativmedizinische Betreuung in } \\
\text { der Praxis, bei Hausbesuchen nach } \\
\text { Nrn. } 01410-01413 \text { oder } 01415\end{array}$ & $\begin{array}{l}35,59 \\
16,59 \\
12,94 \\
12,94\end{array}$ & $\begin{array}{l}\text { zu den links genannten Anlässen in } \\
\text { gleicher Sitzung mit der Nr. } 03370 \\
\text { kombinierbar, nicht aber mit den } \\
\text { Nrn. } 03220 / 03221 \text { sowie } \\
03360 / 03362\end{array}$ \\
\hline
\end{tabular}

möglich. Sämtliche der genannten Nrn. können in jedem Behandlungsfall, also in jedem Quartal einmal angesetzt werden.

Dagegen ist die Palliativleistung nach Nr. 03370, bei der es um die Ersterhebung des $\mathrm{Pa}$ tientenstatus geht, nur einmal im Jahr berechnungsfähig - und dann auch nicht am gleichen Tag wie die Chroniker- und die Geriatrieziffern. Dies gilt auch für die palliativmedizinischen Behandlungsleistungen nach den Nrn. 03371 bis 03373. Ein Ansatz an anderen Tagen beim selben Patienten ist aber hier jeweils möglich.

\section{Letzte Meldung: Software}

Am 5. Februar 2016 haben sich KBV und Kassen auf einen Anforderungskatalog für die Hersteller von Praxisverwaltungssoftware geeinigt. Der Katalog ist Grundlage für die Implementierung eines Zertifizierungsverfahrens für Heilmittel-Verordnungssoftware und beschreibt alle Funktionen, die die Software umsetzen muss. Er ist künftig die verbindliche Grundlage der Heilmittelverordnung durch Vertragsärzte - es kommen hier also neue Entwicklungen auf uns zu. Wir werden darüber berichten.

\section{Hier steht eine Anzeige.}

